\title{
Robust Non-Fragile Control of 2-D Discrete Uncertain Systems: An LMI Approach
}

\author{
Paramanand Sharma, Amit Dhawan \\ Department of Electronics and Communication Engineering, Motilal Nehru National Institute of Technology, Allahabd, India. \\ Email: paramanand84@gmail.com, amit_dhawan2@rediffmail.com
}

Received December $23^{\text {rd }}, 2011$; revised January 31 ${ }^{\text {st }}, 2012$; accepted February $20^{\text {th }}, 2012$

\begin{abstract}
This paper considers the problem of robust non-fragile control for a class of two-dimensional (2-D) discrete uncertain systems described by the Fornasini-Marchesini second local state-space (FMSLSS) model under controller gain variations. The parameter uncertainty is assumed to be norm-bounded. The problem to be addressed is the design of non-fragile robust controllers via state feedback such that the resulting closed-loop system is asymptotically stable for all admissible parameter uncertainties and controller gain variations. A sufficient condition for the existence of such controllers is derived based on the linear matrix inequality (LMI) approach combined with the Lyapunov method. Finally, a numerical example is illustrated to show the contribution of the main result.
\end{abstract}

Keywords: 2-D Discrete Systems; Fornasini-Marchesini Second Local State-Space Model; Non-Fragile Control; Linear Matrix Inequality; Lyapunov Methods

\section{Introduction}

In recent years, the research on two-dimensional (2-D) discrete systems have received considerable attention, since 2-D systems exist in many practical applications such as image data processing, seismographic data processing, thermal processes, gas absorption, water stream heating, river pollution modeling, etc. [1-5]. The stability properties of 2-D discrete systems described by the Fornasini-Marchesini second local state-space (FMSLSS) model [6] have been studied in [7-15]. The asymptotic stability conditions for linear FMSLSS model based on 2-D Lyapunov equation approach have been established in [7-10]. Many publications related to stability analysis of 2-D discrete systems employing various finite wordlength nonlinearities have appeared [10-14]. The problem of robust stability analysis and stabilization of 2-D discrete systems via the linear matrix inequality (LMI) approach has been considered in [15].

Recently, there has been a growing interest in the study of robust non-fragile control problems. The aim of robust non-fragile control is to design a robust controller for a given uncertain system such that the controller is insensitive to some amount of error with regard to its gain. Based on this idea, many significant results have been obtained for one-dimensional case [16-22]. Robust non-fragile control for 2-D discrete uncertain systems in the FMSLSS setting is an important problem.

This paper, therefore, deals with the problem of robust non-fragile control for a class of 2-D discrete uncertain systems described by the FMSLSS model. The paper is organized as follows. In Section 2, we formulate the problem of robust non-fragile control for a class of 2-D discrete uncertain systems described by the FMSLSS model and recall some useful results. The main result of the paper is presented in Section 3. In Section 4, a numerical example is given to illustrate the effectiveness of the proposed method.

Notations $R^{n}$ denotes real vector space of dimension $n, R^{n \times m}$ is the set of $n \times m$ real matrices, 0 denotes null matrix or null vector of appropriate dimension, $\boldsymbol{I}$ is the identity matrix of appropriate dimension, the superscript $T$ stands for matrix transposition, $\boldsymbol{G}>0 \quad(\boldsymbol{G}<0)$ stands for the matrix $\boldsymbol{G}$ is symmetric and positive (negative) definite, and $\operatorname{diag}\{\cdots\}$ stands for a block diagonal matrix.

\section{Problem Formulation and Preliminaries}

This paper studies the problem of robust non-fragile control for a class of 2-D discrete uncertain systems described by the FMSLSS model [6]. Specifically, the system under consideration is given by

$$
\begin{aligned}
& \boldsymbol{x}(i+1, j+1)=\left(\boldsymbol{A}_{1}+\Delta \boldsymbol{A}_{1}\right) \boldsymbol{x}(i+1, j) \\
& \quad+\left(\boldsymbol{A}_{2}+\Delta \boldsymbol{A}_{2}\right) \boldsymbol{x}(i, j+1)+\boldsymbol{B}_{1} \boldsymbol{u}(i+1, j)+\boldsymbol{B}_{2} u(i, j+1),
\end{aligned}
$$

where $\boldsymbol{x}(i, j) \in R^{n}$ and $\boldsymbol{u}(i, j) \in R^{m}$ are the state and 
the control input, respectively. The matrices $\boldsymbol{A}_{1}, \boldsymbol{A}_{2} \in R^{n \times n}$ and $\boldsymbol{B}_{1}, \boldsymbol{B}_{2} \in R^{n \times m}$ are known constant matrices representing the nominal plant. The matrices $\Delta \boldsymbol{A}_{1}$ and $\Delta \boldsymbol{A}_{2}$ represent parameter uncertainties in the system matrices which are assumed to be of the form

$$
\left[\begin{array}{ll}
\Delta \boldsymbol{A}_{1} & \Delta \boldsymbol{A}_{2}
\end{array}\right]=\boldsymbol{L} \boldsymbol{F}(i, j)\left[\begin{array}{ll}
\boldsymbol{M}_{1} & \boldsymbol{M}_{2}
\end{array}\right],
$$

where $\boldsymbol{L} \in R^{n \times k}, \quad \boldsymbol{M}_{1}, \boldsymbol{M}_{2} \in R^{l \times n}$ are known structural matrices of uncertainty and $\boldsymbol{F}(i, j) \in R^{k \times l}$ is an unknown matrix representing parameter uncertainty which satisfies

$$
\begin{gathered}
\boldsymbol{F}^{T}(i, j) \boldsymbol{F}(i, j) \leq \boldsymbol{I} \\
\text { (or equivalently, }\|\boldsymbol{F}(i, j)\| \leq 1) .
\end{gathered}
$$

Suppose the system state is available for feedback, the objective of this paper is to develop a procedure to design a non-fragile state feedback control law

$$
\boldsymbol{u}(i, j)=(\boldsymbol{K}+\Delta \boldsymbol{K}) \boldsymbol{x}(i, j),
$$

where $\boldsymbol{K} \in R^{m \times n}$ is the nominal controller gain and $\Delta \boldsymbol{K}$ represents the controller gain perturbation of the form

$$
\Delta \boldsymbol{K}=\boldsymbol{L}_{k} \boldsymbol{F}_{k}(i, j) \boldsymbol{M}_{k},
$$

with $\boldsymbol{L}_{k} \in R^{m \times g}$ and $\boldsymbol{M}_{k} \in R^{h \times n}$ being known constant matrices, and $\boldsymbol{F}_{k}(i, j) \in R^{g \times h}$ an unknown uncertain parameter matrix satisfying

$$
\begin{gathered}
\boldsymbol{F}_{k}^{T}(i, j) \boldsymbol{F}_{k}(i, j) \leq \boldsymbol{I} \\
\text { (or equivalently, } \left.\left\|\boldsymbol{F}_{k}(i, j)\right\| \leq 1\right),
\end{gathered}
$$

for system (1) such that the resulting closed-loop system

$$
\begin{aligned}
\boldsymbol{x}(i+1, j+1)= & \left(\boldsymbol{A}_{1}+\boldsymbol{B}_{1} \boldsymbol{K}+\Delta \boldsymbol{A}_{1}+\boldsymbol{B}_{1} \Delta \boldsymbol{K}\right) \boldsymbol{x}(i+1, j) \\
& +\left(\boldsymbol{A}_{2}+\boldsymbol{B}_{2} \boldsymbol{K}+\Delta \boldsymbol{A}_{2}+\boldsymbol{B}_{2} \Delta \boldsymbol{K}\right) \boldsymbol{x}(i, j+1)
\end{aligned}
$$

is asymptotically stable for all admissible uncertainties and perturbation in controller gain.

Now, we recall the following lemmas, which are needed in the proof of our main result. As an extension of [7], one can easily arrive at the following lemma.

Lemma 2.1 [7] The system (3) is asymptotically stable if there exists an $n \times n$ positive definite symmetric matrix $\boldsymbol{P}$ such that

$$
\begin{aligned}
& {\left[\begin{array}{lll}
\left(\overline{\boldsymbol{A}}_{1}+\Delta \overline{\boldsymbol{A}}_{1}\right) & \left(\overline{\boldsymbol{A}}_{2}+\Delta \overline{\boldsymbol{A}}_{2}\right)
\end{array}\right]^{T} \boldsymbol{P}\left[\begin{array}{ll}
\left(\overline{\boldsymbol{A}}_{1}+\Delta \overline{\boldsymbol{A}}_{1}\right) & \left.\left(\overline{\boldsymbol{A}}_{2}+\Delta \overline{\boldsymbol{A}}_{2}\right)\right]
\end{array}\right.} \\
& -\left[\begin{array}{cc}
\alpha \boldsymbol{P} & 0 \\
0 & (1-\alpha) \boldsymbol{P}
\end{array}\right]<0 \text {, }
\end{aligned}
$$

for all admissible uncertainties (1b) and (2b) satisfying (1c) and (2c), respectively, where

$$
\begin{gathered}
\overline{\boldsymbol{A}}_{1}=\boldsymbol{A}_{1}+\boldsymbol{B}_{1} \boldsymbol{K}, \overline{\boldsymbol{A}}_{2}=\boldsymbol{A}_{2}+\boldsymbol{B}_{2} \boldsymbol{K}, \\
\Delta \overline{\boldsymbol{A}}_{1}=\Delta \boldsymbol{A}_{1}+\boldsymbol{B}_{1} \Delta \boldsymbol{K}, \Delta \overline{\boldsymbol{A}}_{2}=\Delta \boldsymbol{A}_{2}+\boldsymbol{B}_{2} \Delta \boldsymbol{K}, \\
0<\alpha<1 .
\end{gathered}
$$

Lemma 2.2 [23] Let $\boldsymbol{H}, \boldsymbol{E}, \boldsymbol{F}$ and $\boldsymbol{M}$ be real matrices of appropriate dimension with $\boldsymbol{M}$ satisfying $\boldsymbol{M}=\boldsymbol{M}^{T}$ then

$$
\boldsymbol{M}+\boldsymbol{H F E}+\boldsymbol{E}^{T} \boldsymbol{F}^{T} \boldsymbol{H}^{T}<0
$$

for all $\boldsymbol{F}$ satisfying $\boldsymbol{F}^{T} \boldsymbol{F} \leq \boldsymbol{I}$, if and only if there exists a scalar $\varepsilon>0$ such that

$$
\boldsymbol{M}+\varepsilon \boldsymbol{H} \boldsymbol{H}^{T}+\varepsilon^{-1} \boldsymbol{E}^{T} \boldsymbol{E}<0 .
$$

Lemma 2.3 [24] For real matrices $\boldsymbol{M}, \boldsymbol{L}, \boldsymbol{Q}$ of appropriate dimensions, where $\boldsymbol{M}=\boldsymbol{M}^{T}$ and $\boldsymbol{Q}=\boldsymbol{Q}^{T}>0$ then $\boldsymbol{M}+\boldsymbol{L}^{T} \boldsymbol{Q} \boldsymbol{L}<0$ if and only if

$$
\left[\begin{array}{cc}
\boldsymbol{M} & \boldsymbol{L}^{T} \\
\boldsymbol{L} & -\boldsymbol{Q}^{-1}
\end{array}\right]<0 \text { or equivalently }\left[\begin{array}{cc}
-\boldsymbol{Q}^{-1} & \boldsymbol{L} \\
\boldsymbol{L}^{T} & \boldsymbol{M}
\end{array}\right]<0 \text {. }
$$

\section{Main Result}

In this section, we give a LMI-based sufficient condition for the existence of non-fragile robust controllers in the form of (2a) with the gain perturbation satisfying (2b) and (2c), such that the resulting closed-loop system (3) is asymptotically stable for all admissible parameter uncertainties and controller gain variations.

Theorem 3.1 Consider the system (1a) and the controller gain perturbation $\Delta \boldsymbol{K}$ in (2b) and (2c). Then the robust non-fragile control problem is solvable if there exist positive scalars $\varepsilon_{1}$ and $\varepsilon_{2}$, an $m \times n$ matrix $\boldsymbol{U}$, and an $n \times n$ positive definite symmetric matrix $\boldsymbol{S}$ with a fixed $0<\alpha<1$ such that the following LMI holds:

$$
\left[\begin{array}{c}
\left(-\boldsymbol{S}+\varepsilon_{1} \boldsymbol{L} \boldsymbol{L}^{T}+\varepsilon_{2} \boldsymbol{B}_{1} \boldsymbol{L}_{k} \boldsymbol{L}_{k}^{T} \boldsymbol{B}_{1}^{T}+\varepsilon_{2} \boldsymbol{B}_{2} \boldsymbol{L}_{k} \boldsymbol{L}_{k}^{T} \boldsymbol{B}_{2}^{T}\right) \\
\left(\boldsymbol{A}_{1} \boldsymbol{S}+\boldsymbol{B}_{1} \boldsymbol{U}\right)^{T} \\
\left(\boldsymbol{A}_{2} \boldsymbol{S}+\boldsymbol{B}_{2} \boldsymbol{U}\right)^{T} \\
0 \\
0 \\
0
\end{array}\right.
$$

$$
\left.\begin{array}{cccc}
\left(\boldsymbol{A}_{2} \boldsymbol{S}+\boldsymbol{B}_{2} \boldsymbol{U}\right) & 0 & 0 & 0 \\
0 & \boldsymbol{S M}_{1}^{T} & \boldsymbol{S} \boldsymbol{M}_{k}^{T} & 0 \\
-(1-\alpha) \boldsymbol{S} & \boldsymbol{S} \boldsymbol{M}_{2}^{T} & 0 & \boldsymbol{S} \boldsymbol{M}_{k}^{T} \\
\boldsymbol{M}_{2} \boldsymbol{S} & -\varepsilon_{1} \boldsymbol{I} & 0 & 0 \\
0 & 0 & -\varepsilon_{2} \boldsymbol{I} & 0 \\
\boldsymbol{M}_{k} \boldsymbol{S} & 0 & 0 & -\varepsilon_{2} \boldsymbol{I}
\end{array}\right]<0 .
$$


In this case, a state feedback controller chosen by

$$
\boldsymbol{K}=\boldsymbol{U S}^{-1}
$$

will be such that, for all admissible uncertainties (1b) and (1c), and controller gain variations in (2b) and (2c), the resulting closed-loop system (3) is asymptotically stable.

Proof: Applying Lemma 2.3, (4a) can be written as

$$
\left[\begin{array}{ccc}
-\boldsymbol{P}^{-1} & \left(\overline{\boldsymbol{A}}_{1}+\Delta \overline{\boldsymbol{A}}_{1}\right) & \left(\overline{\boldsymbol{A}}_{2}+\Delta \overline{\boldsymbol{A}}_{2}\right) \\
\left(\overline{\boldsymbol{A}}_{1}+\Delta \overline{\boldsymbol{A}}_{1}\right)^{T} & -\alpha \boldsymbol{P} & 0 \\
\left(\overline{\boldsymbol{A}}_{2}+\Delta \overline{\boldsymbol{A}}_{2}\right)^{T} & 0 & -(1-\alpha) \boldsymbol{P}
\end{array}\right]<0 .
$$

Using (1b), (2b) and (4b), (9) can be represented as

$$
\begin{gathered}
{\left[\begin{array}{ccc}
-\boldsymbol{P}^{-1} & \left(\boldsymbol{A}_{1}+\boldsymbol{B}_{1} \boldsymbol{K}\right) & \left(\boldsymbol{A}_{2}+\boldsymbol{B}_{2} \boldsymbol{K}\right) \\
\left(\boldsymbol{A}_{1}+\boldsymbol{B}_{1} \boldsymbol{K}\right)^{T} & -\alpha \boldsymbol{P} & 0 \\
\left(\boldsymbol{A}_{2}+\boldsymbol{B}_{2} \boldsymbol{K}\right)^{T} & 0 & -(1-\alpha) \boldsymbol{P}
\end{array}\right]+\left[\begin{array}{ccc}
0 & \boldsymbol{L F}(i, j) \boldsymbol{M}_{1} & \boldsymbol{L F}(i, j) \boldsymbol{M}_{2} \\
0 & 0 & 0 \\
0 & 0 & 0
\end{array}\right]+\left[\begin{array}{ccc}
0 & 0 & 0 \\
\boldsymbol{M}_{1}^{T} \boldsymbol{F}^{T}(i, j) \boldsymbol{L}^{T} & 0 & 0 \\
\boldsymbol{M}_{2}^{T} \boldsymbol{F}^{T}(i, j) \boldsymbol{L}^{T} & 0 & 0
\end{array}\right]} \\
+\left[\begin{array}{ccc}
0 & \boldsymbol{B}_{1} \boldsymbol{L}_{k} \boldsymbol{F}_{k}(i, j) \boldsymbol{M}_{k} & \boldsymbol{B}_{2} \boldsymbol{L}_{k} \boldsymbol{F}_{k}(i, j) \boldsymbol{M}_{k} \\
0 & 0 & 0 \\
0 & 0 & 0
\end{array}\right]+\left[\begin{array}{ccc}
0 & 0 & 0 \\
\boldsymbol{M}_{k}^{T} \boldsymbol{F}_{k}^{T}(i, j) \boldsymbol{L}_{k}^{T} \boldsymbol{B}_{1}^{T} & 0 & 0 \\
\boldsymbol{M}_{k}^{T} \boldsymbol{F}_{k}^{T}(i, j) \boldsymbol{L}_{k}^{T} \boldsymbol{B}_{2}^{T} & 0 & 0
\end{array}\right]<0 .
\end{gathered}
$$

Equation (10) can be rewritten as

$$
\begin{aligned}
& {\left[\begin{array}{ccc}
-\boldsymbol{P}^{-1} & \left(\boldsymbol{A}_{1}+\boldsymbol{B}_{1} \boldsymbol{K}\right) & \left(\boldsymbol{A}_{2}+\boldsymbol{B}_{2} \boldsymbol{K}\right) \\
\left(\boldsymbol{A}_{1}+\boldsymbol{B}_{1} \boldsymbol{K}\right)^{T} & -\alpha \boldsymbol{P} & 0 \\
\left(\boldsymbol{A}_{2}+\boldsymbol{B}_{2} \boldsymbol{K}\right)^{T} & 0 & -(1-\alpha) \boldsymbol{P}
\end{array}\right]+\left[\begin{array}{c}
\boldsymbol{L} \\
0 \\
0
\end{array}\right] \boldsymbol{F}(i, j)\left[\begin{array}{lll}
0 & \boldsymbol{M}_{1} & \boldsymbol{M}_{2}
\end{array}\right]+\left[\begin{array}{lll}
0 & \boldsymbol{M}_{1} & \boldsymbol{M}_{2}
\end{array}\right]^{T} \boldsymbol{F}^{T}(i, j)\left[\begin{array}{c}
\boldsymbol{L} \\
0 \\
0
\end{array}\right]^{T}} \\
& +\left[\begin{array}{cc}
\boldsymbol{B}_{1} \boldsymbol{L}_{k} & \boldsymbol{B}_{2} \boldsymbol{L}_{k} \\
0 & 0 \\
0 & 0
\end{array}\right]\left[\begin{array}{cc}
\boldsymbol{F}_{k}(i, j) & 0 \\
0 & \boldsymbol{F}_{k}(i, j)
\end{array}\right]\left[\begin{array}{ccc}
0 & \boldsymbol{M}_{k} & 0 \\
0 & 0 & \boldsymbol{M}_{k}
\end{array}\right] \\
& +\left[\begin{array}{ccc}
0 & \boldsymbol{M}_{k} & 0 \\
0 & 0 & \boldsymbol{M}_{k}
\end{array}\right]^{T}\left[\begin{array}{cc}
\boldsymbol{F}_{k}(i, j) & 0 \\
0 & \boldsymbol{F}_{k}(i, j)
\end{array}\right]^{T}\left[\begin{array}{cc}
\boldsymbol{B}_{1} \boldsymbol{L}_{k} & \boldsymbol{B}_{2} \boldsymbol{L}_{k} \\
0 & 0 \\
0 & 0
\end{array}\right]^{T}<0 .
\end{aligned}
$$

Using Lemma 2.2, (11) can be rearranged as

$$
\left[\begin{array}{ccc}
\left(-\boldsymbol{P}^{-1}+\varepsilon_{1} \boldsymbol{L} \boldsymbol{L}^{T}+\varepsilon_{2} \boldsymbol{B}_{1} \boldsymbol{L}_{k} \boldsymbol{L}_{k}^{T} \boldsymbol{B}_{1}^{T}+\varepsilon_{2} \boldsymbol{B}_{2} \boldsymbol{L}_{k} \boldsymbol{L}_{k}^{T} \boldsymbol{B}_{2}^{T}\right) & \left(\boldsymbol{A}_{1}+\boldsymbol{B}_{1} \boldsymbol{K}\right) & \left(\boldsymbol{A}_{2}+\boldsymbol{B}_{2} \boldsymbol{K}\right) \\
\left(\boldsymbol{A}_{1}+\boldsymbol{B}_{1} \boldsymbol{K}\right)^{T} & -\alpha \boldsymbol{P}+\varepsilon_{1}^{-1} \boldsymbol{M}_{1}^{T} \boldsymbol{M}_{1}+\varepsilon_{2}^{-1} \boldsymbol{M}_{k}^{T} \boldsymbol{M}_{k} & \varepsilon_{1}^{-1} \boldsymbol{M}_{1}^{T} \boldsymbol{M}_{2} \\
\left(\boldsymbol{A}_{2}+\boldsymbol{B}_{2} \boldsymbol{K}\right)^{T} & \varepsilon_{1}^{-1} \boldsymbol{M}_{2}^{T} \boldsymbol{M}_{1} & -(1-\alpha) \boldsymbol{P}+\varepsilon_{1}^{-1} \boldsymbol{M}_{2}^{T} \boldsymbol{M}_{2}+\varepsilon_{2}^{-1} \boldsymbol{M}_{k}^{T} \boldsymbol{M}_{k}
\end{array}\right]<0 .
$$

Premultiplying and postmultiplying (12) by $\operatorname{diag}\left\{\boldsymbol{I}, \boldsymbol{P}^{-1}, \boldsymbol{P}^{-1}\right\}$, one obtains

$$
\begin{gathered}
{\left[\begin{array}{ccc}
\left(-\boldsymbol{S}+\varepsilon_{1} \boldsymbol{L} \boldsymbol{L}^{T}+\boldsymbol{\varepsilon}_{2} \boldsymbol{B}_{1} \boldsymbol{L}_{k} \boldsymbol{L}_{k}^{T} \boldsymbol{B}_{1}^{T}+\boldsymbol{\varepsilon}_{2} \boldsymbol{B}_{2} \boldsymbol{L}_{k} \boldsymbol{L}_{k}^{T} \boldsymbol{B}_{2}^{T}\right) & \left(\boldsymbol{A}_{1} \boldsymbol{S}+\boldsymbol{B}_{1} \boldsymbol{U}\right) & \left(\boldsymbol{A}_{2} \boldsymbol{S}+\boldsymbol{B}_{2} \boldsymbol{U}\right) \\
\left(\boldsymbol{A}_{1} \boldsymbol{S}+\boldsymbol{B}_{1} \boldsymbol{U}\right)^{T} & -\alpha \boldsymbol{S} & 0 \\
\left(\boldsymbol{A}_{2} \boldsymbol{S}+\boldsymbol{B}_{2} \boldsymbol{U}\right)^{T} & 0 & -(1-\alpha) \boldsymbol{S}
\end{array}\right]} \\
+\left[\begin{array}{ccc}
0 & 0 & 0 \\
0 & \varepsilon_{1}^{-1} \boldsymbol{S} \boldsymbol{M}_{1}^{T} \boldsymbol{M}_{1} \boldsymbol{S}+\boldsymbol{\varepsilon}_{2}^{-1} \boldsymbol{S} \boldsymbol{M}_{k}^{T} \boldsymbol{M}_{k} \boldsymbol{S} & \varepsilon_{1}^{-1} \boldsymbol{S} \boldsymbol{M}_{1}^{T} \boldsymbol{M}_{2} \boldsymbol{S} \\
0 & \varepsilon_{1}^{-1} \boldsymbol{S} \boldsymbol{M}_{2}^{T} \boldsymbol{M}_{1} \boldsymbol{S} & \varepsilon_{1}^{-1} \boldsymbol{S} \boldsymbol{M}_{2}^{T} \boldsymbol{M}_{2} \boldsymbol{S}+\varepsilon_{2}^{-1} \boldsymbol{S} \boldsymbol{M}_{k}^{T} \boldsymbol{M}_{k} \boldsymbol{S}
\end{array}\right]<0,
\end{gathered}
$$

where $\boldsymbol{S}=\boldsymbol{P}^{-1}$ and $\boldsymbol{K}=\boldsymbol{U} \boldsymbol{S}^{-1}$.

The equivalence of (13) and (7) follows trivially from Lemma 2.3. This completes the proof of Theorem 3.1.

\section{Numerical Example}

As an illustration of Theorem 3.1, consider a 2-D discrete uncertain system represented by (1) with 


$$
\begin{gathered}
\boldsymbol{A}_{1}=\left[\begin{array}{ll}
0 & 0 \\
1 & 0
\end{array}\right], \quad \boldsymbol{A}_{2}=\left[\begin{array}{cc}
0.1 & 1 \\
0 & 0
\end{array}\right], \quad \boldsymbol{B}_{1}=\left[\begin{array}{l}
0.002 \\
0.002
\end{array}\right], \\
\boldsymbol{B}_{2}=\left[\begin{array}{c}
-0.008 \\
0
\end{array}\right], \quad \boldsymbol{L}=\left[\begin{array}{l}
0 \\
1
\end{array}\right], \quad \boldsymbol{M}_{1}=\left[\begin{array}{ll}
0.7 & -0.008
\end{array}\right], \\
\boldsymbol{M}_{2}=\left[\begin{array}{ll}
0.5 & -0.02
\end{array}\right] .
\end{gathered}
$$

Suppose the actual controller is with perturbations in the form of (2b) and (2c) with parameters as

$$
\boldsymbol{L}_{k}=0.15, \quad \boldsymbol{M}_{k}=\left[\begin{array}{ll}
0 & 0.02
\end{array}\right] \text {. }
$$

We wish to design a robust non-fragile state feedback controller for this system such that the resulting closedloop system is asymptotically stable for all admissible uncertainties and controller gain variations. By solving LMI (7) using the Matlab LMI toolbox [24,25] with $\alpha=0.7$, we obtain the following feasible solution:

$$
\begin{gathered}
\boldsymbol{S}=\left[\begin{array}{cc}
2.4802 & 0.2958 \\
0.2958 & 18.1199
\end{array}\right], \varepsilon_{1}=7.1774, \varepsilon_{2}=7.6242 \\
\boldsymbol{U}=\left[\begin{array}{ll}
70.8 & 2140.3
\end{array}\right] .
\end{gathered}
$$

Therefore, by Theorem 3.1, we can see that the robust non-fragile control problem is solvable. A desired state feedback controller to solve this problem can be chosen as

$$
\boldsymbol{K}=\left[\begin{array}{ll}
14.4699 & 117.8843
\end{array}\right]
$$

\section{Conclusion}

In this paper, we have investigated the problem of robust non-fragile control for a class of 2-D discrete uncertain systems in the FMSLSS setting under state feedback gain variations. Using the Lyapunov method, a criterion for robust non-fragile control via state feedback is derived in terms of LMI. Finally, a numerical example has been presented to illustrate the effectiveness of the proposed method.

\section{REFERENCES}

[1] T. Kaczorek, "Two-Dimensional Linear Systems," SpringerVerlag, Berlin, 1985.

[2] R. N. Bracewell, “Two-Dimensional Imaging,” PrenticeHall, Englewood, 1995, pp. 505-537.

[3] W.-S. Lu and A. Antoniou, "Two-Dimensional Digital Filters,” Marcel Dekker, New York, 1992.

[4] N. K. Bose, "Applied Multidimensional system Theory,” Van Nostrand Reinhold, New York, 1982.

[5] E. Fornasini, “A 2-D System Approach to River Pollution Modeling," Multidimensional Systems and Signal Processing, Vol. 2, No. 3, 1991, pp. 233-265. doi:10.1007/BF01952235

[6] E. Fornasini and G. Marchesini, "Doubly Indexed Dy- namical Systems: State-Space Models and Structural Properties,” Theory of Computing Systems, Vol. 12, No. 1, 1978, pp. 59-72.

[7] T. Hinamoto, "2-D Lyapunov Equation and Filter Design Based on the Fornasini-Marchesini Second Model,” IEEE Transactions on Circuits and Systems I, Vol. 40, No. 2, 1993, pp. 102-110.

[8] W.-S. Lu, "On a Lyapunov Approach to Stability Analysis of 2-D Digital Filters," IEEE Transactions on Circuits and Systems I, Vol. 41, No. 10, 1994, pp. 665-669. doi:10.1109/81.329727

[9] T. Ooba, "On Stability Analysis of 2-D Systems Based on 2-D Lyapunov Matrix Inequalities,” IEEE Transactions on Circuits and Systems I, Vol. 47, No. 8, 2000, pp. 12631265. doi:10.1109/81.873883

[10] T. Hinamoto, "Stability of 2-D Discrete Systems Described by the Fornasini-Marchesini Second Model," IEEE Transactions on Circuits and Systems I, Vol. 34, No. 2, 1997, pp. 254-257. doi:10.1109/81.557373

[11] D. Liu, "Lyapunov Stability of Two-Dimensional Digital Filters with Overflow Nonlinearities," IEEE Transactions on Circuits and Systems I, Vol. 45, No. 5, 1998, pp. 574577. doi:10.1109/81.668870

[12] H. Kar and V. Singh, "An Improved Criterion for the Asymptotic Stability of 2-D Digital Filters Described by the Fornasini-Marchesini Second Model Using Saturation Arithmetic," IEEE Transactions on Circuits and Systems I, Vol. 46, No. 11, 1999, pp. 1412-1413. doi:10.1109/81.802847

[13] H. Kar and V. Singh, "Stability Analysis of 2-D Digital Filters Described by the Fornasini-Marchesini Second Model Using Overflow Nonlinearities," IEEE Transactions on Circuits and Systems I, Vol. 48, No. 5, 2001, pp. 612-617. doi:10.1109/81.922464

[14] H. Kar and V. Singh, "Stability Analysis of 1-D and 2-D Fixed-Point State-Space Digital Filters Using Any Combination of Overflow and Quantization Nonlinearities,” IEEE Transactions on Signal Processing, Vol. 49, No. 5, 2001, pp. 1097-1105. doi:10.1109/78.917812

[15] C. Du and L. Xie, "Stability Analysis and Stabilization of Uncertain Two-Dimensional Discrete Systems: An LMI Approach,” IEEE Transactions on Circuits and Systems I, Vol. 46, No. 11, 1999, pp. 1371-1374. doi:10.1109/81.802835

[16] W. M. Haddad and J. R. Corrado, "Robust Resilient Dynamic Controllers for Systems with Parameter Uncertainty and Controller Gain Variations,” International Journal of Control, Vol. 73, 2000, pp. 1405-1423. doi:10.1080/002071700445424

[17] G.-H. Yang, J. L. Wang and C. Lin, “ $H_{\infty}$ Control for Linear Systems with Additive Controller Gain Variations," International Journal of Control, Vol. 73, No. 16, 2000, pp. 1500-1506. doi:10.1080/00207170050163369

[18] G.-H. Yang and J. L. Wang, "Non-Fragile $H_{\infty}$ Control for Linear Systems with Multiplicative Controller Gain Variations,” Automatica, Vol. 37, No. 5, 2001, pp. 727737.

[19] J. H. Park, "Robust Non-Fragile Control for Uncertain 
Discrete-Delay Large-Scale Systems with a Class of Controller Gain Variations," Applied Mathematics and Computation, Vol. 149, No. 1, 2004, pp. 147-164. doi:10.1016/S0096-3003(02)00962-1

[20] S. Xu, J. Lam, J. Wang and G. Yang, "Non-Fragile Positive Real Control for Uncertain Linear Neutral Delay Systems," Systems and Control Letters, Vol. 52, No. 1, 2004, pp. 59-74. doi:10.1016/j.sysconle.2003.11.001

[21] C. Lien, W. Cheng, C. Tsai and K. Yu, "Non-Fragile Observer-Based Controls of Linear System via LMI Approach,” Chaos, Solitons and Fractals, Vol. 32, No. 4 2007, pp. 1530-1537. doi:10.1016/j.chaos.2005.11.092

[22] C. Lien, “ $H_{\infty}$ Non-Fragile Observer-Based Controls of Dynamical Systems via LMI Optimization Approach,”
Chaos, Solitons and Fractals, Vol. 34, No. 2, 2007, pp. 428-436. doi:10.1016/j.chaos.2006.03.050

[23] L. Xie, "Output Feedback $H_{\infty}$ Control of Systems with Parameter Uncertainty,” International Journal of Control, Vol. 63, No. 4, 1996, pp. 741-750. doi:10.1080/00207179608921866

[24] S. Boyd, L. El Ghaoui, E. Feron and V. Balakrishnan, "Linear Matrix Inequalities in System and Control Theory,” SIAM, Philadelphia, 1994. doi:10.1137/1.9781611970777

[25] P. Gahinet, A. Nemirovski, A. J. Laub and M. Chilali, "LMI Control Toolbox-for Use with Matlab," The MATH Works Inc., Natick, 1995. 